\title{
Plane jets impinging on porous walls
}

\author{
Ross $\mathrm{CANT}^{1,2 *}$ Ian $\mathrm{CASTRO}^{1 \dagger}$ and Peter WALKLATE ${ }^{2}$ \\ ${ }^{1}$ School of Mechanical \& Materials Engineering \\ University of Surrey, Guildford, Surrey, GU2 5XH, UK \\ ${ }^{2}$ Process Engineering Division, Silsoe Research Institute \\ Silsoe, Bedford, MK45 4HS
}

Contact e-mail:i.castro@soton.ac.uk

The flow of a two-dimensional, plane turbulent jet impinging on a porous screen has been studied experimentally. It is shown how the overall flow structure depends on the porosity of the surface. For low screen porosity $(\beta<0.41$, say), transverse wall jets can be formed on both sides of the screen and in extreme cases the axial momentum flux some way downstream of the screen falls to zero, so that the screen has the same drag as would a solid wall. For high screen porosity $(\beta>0.57$, say) the axial volume flux is largely preserved through the screen but the dominant eddy structures present in the upstream jet are largely destroyed so that entrainment rates downstream of the screen can be very low. The relatively small, intermediate range of porosities $(0.41<\beta<0.57$, where $\beta$ is the screen open area ratio), is associated with dramatic changes in flow pattern and recirculating regions can exist on the upstream side of the screen. These flows, although all geometrically very simple, provide a serious challenge for computational modelling.

\section{Introduction}

Two-dimensional plane jets are a classical type of inhomogeneous turbulent shear flow and their mean flow and turbulent structure is well known (e.g. Gutmark \& Wygnanski, 1976, Everitt \& Robins, 1978, etc.). Likewise, axisymmetric jets and also their impingement onto a solid surface have been widely studied, the latter often in the context of VSTOL (Vertical Short Takeoff and Landing) applications. In contrast, there are few published studies of planar jets impinging on solid surfaces - Beltaos \& Rajaratnam (1973) (hereafter denoted by $\mathrm{BR}$ ) is a rare example. In this case, the jet is turned through $90^{\circ}$ by the solid surface and eventually develops into two planar wall jets moving in opposite directions. In the better-known axi-symmetric case a radial wall jet is produced (e.g. Foss, 1979, Özdemir \& Whitelaw, 1992).

There are some practical situations in which the impingement surface is not solid. The present work formed part of a more extensive study of jets impinging on porous regions, with the particular application of orchard crop-spraying in mind. In this instance, the sprayer typically produces an air jet to enhance the transport and deposition of pesticide spray throughout the vegetative volume of large bush and tree crops. There has been some study of the nature of the penetration of such jets and how the flow patterns indicate different mechanisms for describing the surface interface layer and inner volume of a generic

\footnotetext{
*Present address: M \& N Wind Power Ltd, 15e Cuxhaven Way, Longrock, Penzance TR20 8HX, UK

${ }^{\dagger}$ Present address: School of Engineering Sciences, University of Southampton, Highfield, Southampton, SO17 1BJ, UK
} 
porous structure (Weiner, 1993, Walklate et al 1996, Walklate \& Weiner, 1997). Simple scaling properties of the jet penetration of a porous volume have been derived from the analytical solution of the governing two-dimensional momentum equation (Walklate et al, 1996). However, the flow and momentum redistribution due to interaction of the jet and the outer surface of a porous structure is less well understood and the present idealised experiment was conceived in order to shed some light on this aspect of the problem.

The nature of the jet penetration through both the initial row of obstacles and the later rows must clearly depend on the scale of the obstacles and spaces between them (the 'scale of the porosity') compared with the scale of the jet. Previous work has shown that if the jet has a width (at the porous surface location but in its absence) of the same order as the porosity scale, then it can bifurcate, with different 'branches' of the jet moving through adjacent spaces between the solid obstacles (Weiner, 1993). In these circumstances, details of the penetration behaviour, and of the interaction with the first row of obstacles, depend strongly on the precise geometry of the porosity. On the other hand, if the porosity scale is small compared with the jet scale then one would not expect such bifurcating behaviour and, in the limit of small enough porosity scale, the penetration details should presumably not depend on the details of the porosity but only on the overall parameter defining the porosity itself - here taken as $\beta$, the open area ratio (i.e. the ratio of open area to solid area of the screen). (Of course, even for a uniform upstream flow, in a region just downstream of the screen there will always be individual jets corresponding to flow through the 'holes', whatever the shape of the latter, and these jets will subsequently coalesce, perhaps unstably, depending on the screen porosity - Bradshaw, 1965). We concentrate here on the idealised case of an upstream jet whose width is large compared to the geometry of the porosity, studying only the flow behaviour for jet impingement onto a single, thin screen but with various values of $\beta$. This can be thought of as an idealisation of the flow at the interface between a jet and a porous volume.

The experiments are in some senses preliminary. No comprehensive attempt has been made to examine the details of the turbulent structure either in the impingement region or downstream of the screen. Attention is concentrated on the influence of the porosity, $\beta$, on the overall nature of the flow, so as to delineate the ranges of $\beta$ that give rise to fundamentally different flows. Nonetheless, some of the turbulence measurements are used where necessary to assist in explaining the characteristics of the different flow types.

\section{Experimental Arrangement}

The (nominally) two-dimensional plane jet was produced by fitting an additional contraction to the $300 \times 600 \mathrm{~mm}$ contraction exit of an open-circuit blower tunnel in the EnFlo laboratory at the University of Surrey. The final outlet was $600 \mathrm{~mm}$ wide by $5.5 \mathrm{~mm}$ and side walls were installed to confine the spreading of the jet to the transverse $y$-direction only. One of these walls was perspex to allow penetration of laser doppler anemometry beams. Figure 1 shows the general arrangement and a full description of the rig is given by Guridi (1997).

For most experiments the outlet velocity, $U_{o}$, was maintained at about $40 \mathrm{~m} / \mathrm{s}$, giving an outlet jet Reynolds number based on total jet width $\left(b_{o}\right)$ of about 15000 . Initial checks showed that the outlet flow was highly uniform in the spanwise direction and had a closely 'top-hat' velocity profile, with extremely thin boundary layers. The outlet velocity was monitored by a $1.5 \mathrm{~mm}$ o.d. total head tube connected to a standard micromanometer.

The porous screens were constructed from $0.9 \mathrm{~mm}$ thick sheets of perforated steel, with circular holes uniformly spaced on a $60^{\circ}$ triangular grid. Four screens were used, having hole diameter and pitch, respectively, of $(12.7,15),(12.7,16),(9.5,12.8)$ and $(6.4,9.5)$, with all 
dimensions in $\mathrm{mm}$. These values give porosities, $\beta$, of $0.65,0.57,0.5$ and 0.41 , respectively. The maximum pitch $(16 \mathrm{~mm})$ ensured that there were at least 15 holes across the width of the jet at the screen location, defined by $H=x_{s}=500 \mathrm{~mm}$. This was deemed sufficient to ensure that the porosity geometry would not be a significant parameter in determing the flow. (Some earlier tests conducted at Silsoe Research Institute lend support to this assumption - see Cant, 1999). In addition to this criterion, the screen location and (jet) nozzle size were chosen to ensure that the free jet would achieve its self-preserving state before the influence of the screen would be felt.

All the mean velocity and turbulence measurements in the jets were made using a onecomponent (Dantec) laser Doppler anemometer. The optics produced two beams focussed through a front lens of $500 \mathrm{~mm}$ focal length, resulting in a measurement volume of $0.24 \mathrm{~mm}$ diameter and about $6 \mathrm{~mm}$ long, the long axis being aligned with the spanwise $(z)$ direction. Resulting errors arising from spatial averaging in the spanwise direction were insignificant because of the relatively large scale of the jet flow at most measurement locations. The probe was mounted on a manual traverse gear located to the side of the jet (outside the perspex side wall). This allowed positioning in $1 \mathrm{~mm}$ increments through the ranges $0<x<900 \mathrm{~mm}$ and $-400<y<400 \mathrm{~mm}$. All data presented here were obtained in the central plane of the jet $-z=0$. The photomultiplier signal was analysed using a burst detector and a covariance processor operated by the Dantec PC-based software systems.

Seeding, in the form of silicon oil droplets produced by a commercial smoke generator, was delivered into the jet via both the jet air itself (injecting upstream of the blower) and the entrainment air (by using a spanwise, 'multi-hole' pipe suitably positioned in the far field). Particle sizing of the smoke, undertaken using a Dantec Particle Phase Doppler Analyser (PDA), showed that $99.8 \%$ of the particles had diameters below $5 \mu \mathrm{m}$ which, according to the criterion that the particle time constant should be less than the Kolmogorov timescale (George \& Taulbee, 1992), was more than adequate in this flow. Velocity bias arising from the entrained air containing no particles was obviated by seeding this air as well as the primary jet air, as explained earlier. To prevent any possible velocity bias arising from non-periodic sampling - more fast particles arriving than slow, per unit time - the dead-time facility in the sampling software was set to $0.05 \mathrm{~s}$, which resulted in a nearly uniform sampling rate of about $20 \mathrm{~Hz}$. For the spectral measurements, the sampling rate was about $40 \mathrm{~Hz}$. Preliminary tests were carried out using a range of settings for the photomultiplier voltage, velocity range (via the bandwidth) and gain, in order to achieve the maximum possible data rate without compromising the accuracy of the results because of spurious values in the velocity histogram. We estimate, partly on the basis of these tests, that mean velocity and turbulence (standard deviation) data are accurate to within at least $2 \%$ of $U_{o}$ and usually significantly better. Certainly the repeatability is consistently much better than this.

\section{Results and Discussion}

\subsection{The Free Jet}

Mean velocity and turbulence data taken in the unobstructed jet had the expected features. Figure 2 presents mean velocity data obtained at various downstream locations, normalised by the maximum value, $U_{m}$, at each location and with the spanwise variable normalised by the jet width, $b$, defined as the value of $y$ where the mean velocity was $0.5 U_{m}$. The profiles have the usual Gaussian behaviour and are closely self-similar. Figure 3 shows the growth of the jet width and the decay of the centreline velocity and includes the linear best fit lines, forced to give the same virtual origin. Slightly improved fits would be possible, particularly to the $U_{m}$ data, if this latter condition were relaxed but, nevertheless, the 
slopes are close to those found in the literature. In the present case, the slopes are $(0.103$, $0.185)$ for the jet width and inverse velocity relations, respectively - see fig. 3 - and can be compared with those found by, for example, BR (0.100, 0.174), Flora \& Goldschmidt (1969) $(0.13,0.23)$ and Gutmark \& Wygnanski (1976) $(0.100,0.175)$. Everitt \& Robins (1978) had a range of results $(0.09-0.11,0.14-0.22)$ but their jets included those with a co-flowing stream. Note that the data at the final downstream station have not been included in the fits in fig. 3 and it is clear that there is some departure from linearity beyond about $x / b_{o}=135$. This is probably a result of residual draughts in the laboratory; $U_{m}$ at this location was only about $6 \mathrm{~m} / \mathrm{s}$. Axial turbulence intensity data are shown in Figure 4 . Close similarity is again observed, with the data lying between that of Gutmark \& Wygnanski (1976) and Everitt \& Robins (1978).

It is worth noting that in common with most experiments on turbulent jets, the exit momentum flux, $M_{o}$, is somewhat higher than that calculated from the downstream velocity profiles, $M(x)$. In fact, $M(x) / M_{o}$, gradually falls with increasing $x$, to about 0.8 at $x / b_{o}=155$. This behaviour has been observed in previous work and Schneider (1985) showed it to be a result of the recirculating flow inevitably present for a rig which takes fluid from a closed environment (e.g. inlet air to a blower) and returns the same fluid to it (via the jet itself). It is of no major concern in the present case since our essential intention is to study the changes in the jet flow caused by the imposition of a porous screen.

\subsection{Jet impingement on a solid wall}

The free jet is one of the two limiting cases and corresponds to $\beta=1$. Before presenting data for the porous surface impingement cases, the results for the other limiting case (the solid wall, $\beta=0$ ) are briefly discussed. Figures 5 and 6 show mean velocity and turbulence intensity profiles, normalised in the same way as the data in figures 2 and 4 . In this case the profiles are distinguished by their $x / H$ values in addition to their $x / b_{o}$ values. $H$ is the axial position of the screen (and note that $H=91 b_{o}$ ). The mean velocity profiles appear to remain self-similar, following the free jet data, up to at least $x / H=0.9$, but by that stage both the jet width and the centre-line velocity are significantly different from the free jet values. Their variations are included in Figure 3 and it is evident that $b / b_{o}$ and $\left(U_{o} / U_{m}\right)^{2}$ begin to deviate from their free jet values around $x / b_{o}=70(x / H=0.7)$. Figure 6 shows that the axial turbulence intensities begin to rise above the free jet values for $x / H>0.8$, so the 'apparent' self-similarity of the turbulence evidently disappears a little earlier than for the mean flow. The ratio $H / b_{o}$ is different in the present experiments from that used by BR. However, normalising the axial location by $H$ and the jet width and centreline velocity by the values they would have in the free jet allows the two sets of data to be compared. This is done in Figure 7 . For both jet properties it is clear that the jet behaves essentially as a free jet up to around $x / H=0.7$, beyond which both the centreline velocity decrease and the jet width increase is more rapid than in the free jet. There is reasonable agreement between the two experiments. Once the jet completes its $90^{\circ}$ turn a wall jet develops and Figure 8 shows the resulting velocity profiles at various distances from the original jet centreline. The data are normalised using the the peak velocity, $V_{m}$, and the distance, $b_{x}$, from the wall to the point where the velocity is $0.5 V_{m}$. It is evident that there is a good degree of similarity, at least over the $y / H$ range shown. Wygnanski et al's (1992) data are included for comparison; whilst agreement is reasonable, the differences are not insignificant and are almost certainly due to the different means of producing the wall jet. Wygnanski et al used the classical arrangement - tangential blowing from an orifice attached to the wall. In the present case one might expect the wall jet to take a long time to recover from the complex effects of the mean strains in the impingement region. 
It is concluded from all the previous results that in both the limiting cases $(\beta=0$ and $\beta=1$ ) the flows have the behaviour expected on the basis of the previous literature. We turn now to the results obtained with the solid wall replaced by the various porous screens.

\subsection{Porous screen cases}

Mean velocity and turbulence profiles were obtained for the four (non-zero porosity) porous screen cases $(\beta=0.65,0.57,0.5$ and 0.41$)$ and used to obtain the jet growth, the maximum velocity decay and the axial volume and momentum fluxes at each axial location, including a number of locations downstream of the screen (up to $x / H=1.7$ ). We discuss first the mean flow properties. As might be anticipated from a comparison of figs. 2 and 5 , the mean velocity profiles in each case were reasonably self-similar, even for those profiles obtained beyond the screen, and so are not shown here.

Figures 9 and 10 show the decay of the centre-line velocity and the growth in jet width, respectively, for each case. The behaviour is clearly consistent with the overall expectation that the downstream jet becomes progressively more diffuse as the screen porosity decreases. In every case the jet width is increased by the screen, with a corresponding fall in the maximum velocity below the free jet value. However, there are significant differences between the various cases. For $\beta=0.65$ and 0.57 , after the initial increase in the region of the screen itself, the growth rate is substantially reduced (fig. 10); indeed, the data suggest that the jet ceases to grow altogether. We return to this point later. On the other hand, for $\beta=0.5$ the jet width continues to grow rapidly, at least up to about $x / H=1.4$ and there is a corresponding continual fall in centre-line velocity (fig. 9). In the $\beta=0.4$ case this fall in velocity is even more rapid and, in fact, the velocity reaches zero around $x / H=1.3$. Few definitive measurements were made further downstream in this case but some isolated tests indicated that the velocity did become negative, although very small in magnitude, as the data in fig. 9 would suggest. It was difficult to determine jet width in this very-low-velocity region so that the single data point at $x / H=1.3$ for $\beta=0.41$ is probably in error.

These results suggest qualitative differences in the overall flow structure in different $\beta$ ranges. Figures 11 and 12, which show the (axial) volume and momentum fluxes calculated by appropriate integrations of the mean velocity data, emphasise these differences. A number of points should be noted. First, as indicated earlier, the free jet momentum flux falls slowly with $x$ (fig.12). The screen drag leads to a fairly sudden fall in momentum, by an amount which depends on $\beta$, as expected. However, except at the lowest (non-zero) porosity the decay in momentum flux downstream of the screen recovers roughly to the rate seen in the free jet. Secondly, for this $\beta=0.41$ case the screen effectively removes all the axial momentum. Thirdly, downstream of the screen the volume flux (fig.11) actually falls with $x$ for the two more solid screens $(\beta=0.5$ and 0.41$)$, whilst remaining virtually constant for the two more open cases $(\beta=0.57$ and 0.65$)$. A falling volume flux must be a consequence of the existence of significant wall jets transporting fluid out sideways away from the impingement zone (as for the $\beta=0$ case). Figure 13, which shows measurements of the lateral mean velocity at three fixed $y / H$ locations for the $\beta=0.41$ (fig. 13a) and the $\beta=0.5$ (fig. 13b) cases, confirms the existence of such wall jets. For $\beta=0.5$ the jet is relatively weak, with the peak $V / U_{o}$ (at $y / H=0.4$ ) reaching only about 0.06 compared with 0.14 in the $\beta=0.41$ case, and it seems not to survive beyond about $y / H=0.5$. Wall jets did not exist at all for the more open screens.

Consideration of the likely pressure field also suggests that such wall jets, on both sides of the screen, must exist for sufficiently low porosity. There will be a static pressure rise upstream of the screen - driving wall jets on that side - and although there will then be a significant (local) pressure drop across the screen, the static pressure just downstream must 
still, for low enough porosity, be initially significantly higher than atmospheric so that wall jets could also be driven on the downstream side. When the porosity is higher, the static pressure levels near the screen (on $y=0$ ) will not be sufficient to initiate wall jets. This seems to be the situation for the $\beta=0.57$ and 0.65 cases. Figure 14 shows the transverse mean velocity variation along the line $y=2.5 b$ - near the edge of the jet. Sufficiently far upstream of the screen the $V$-component is negative, being essentially a measure of the entrainment process. As the screen is approached, however, $V$ rises rapidly, indicating the turning of the jet. For the two more solid (but porous) cases, $V$ remains positive downstream of the screen, because of the wall jets present on that side also, whereas for $\beta=0.57$ it returns to negative values, indicating that the jet is continuing in the downstream $x$-direction. If the porosity is sufficiently low to give strong wall jets on both sides of the screen (as in the $\beta=0.41$ case) entrainment into the wall jet on the downstream side of the screen would induce a flow in the -ve $x$-direction further downstream and this could account for the small negative volume and (possibly) momentum fluxes shown in figs. 11 and 12. However, a negative momentum flux implies that the screen drag coefficient is actually higher than it would be for a solid wall, which seems intuitively unlikely. On the other hand, fig.13(a) suggests that the downstream wall jet does not grow significantly with increasing distance from the impingement zone, in contrast to the upstream wall jet whose velocity profiles look similar to those obtained for the solid wall case. So any entrainment velocity would be extremely small. In addition there could be weak lateral (and spanwise) flows near the edges of the screens which, given also the rather uncertain influence of recirculating laboratory air, would make the execution of a complete momentum balance rather uncertain. Such balances would strictly, of course, need to include the turbulence contributions and these might be quite significant far downstream (see below).

What is certain, however, is that at least not too far from $y=0$ there is an upstream flow far downstream of the highest solidity screen. This is emphasised by the streamline patterns deduced by appropriate integrations of the velocity data and sketched in Figure 15. In each case the region shown covers the ranges $0<x / H<1.7$ and $-0.8<y / H<0.8$. The different nature of the mean flow for the different degrees of porosity is clear, with the higher porosity screens leading to an increase in jet width and the lowest porosity leading to wall jets on both sides with inflow from far downstream. The intermediate case, $\beta=0.5$, gives what might be termed a 'fan jet' downstream of the screen. Notice too that in this case there is evidence of recirculating regions on the upstream side of the screen. This may also occur for the $\beta=0.41$ case but, if so, it is clearly on a considerably larger scale and might thus be more influenced by far field effects (arising from the finite geometry of the rig). To confirm the overall mean flow behaviour for different levels of screen porosity some flow visualisation experiments were undertaken. These used a different rig, at Silsoe Research Institute, but the results (see Cant, 1999) were entirely consistent with the more quantitative data presented here.

We complete our presentation of the measurements by considering some of the turbulence data and their implications. Figure 16 shows a selection of the axial rms turbulence profiles (normalised by the local maximum velocity). Data for $x / H<0.7$ are not shown as they collapse well to the free jet results (as noted for the $\beta=0$ case shown in fig. 6). Note first that for the two most open cases (figs. 16a and 16b) the screen acts to reduce the turbulence energy below the free jet values, after an initial increase just behind it. This latter increase is noticeably larger for $\beta=0.57$ than for $\beta=0.65$ and for lower porosities the reduction to energy levels lower than free jet values does not occur, although for $\beta=0.5$ there is an initial fall to (almost) the free jet value before a relatively slow rise further downstream. The behaviour is summarised in Figure 17, which shows the centre-line intensity variation 
for all cases. Of course, when normalised by a fixed velocity scale (like the jet exit velocity, $U_{o}$ ), rather than the local maximum velocity as in the figure $17 \mathrm{a}$, all the screens act to reduce the turbulence energy levels substantially; this is shown in fig. 17b. At $x / H=1.3$, for example, $u^{\prime} / U_{o}$ is in all cases less than about one half of the free jet value at the same location. But the data (in fig. 17a) are clearly consistent with the earlier conclusion that there is a distinct change in flow structure between the $\beta=0.41$ case and the two more open screens, with the $\beta=0.5$ case having an intermediate behaviour.

\section{Further Results and Final Discussion}

The results presented in the previous sections have led to a reasonably clear picture of the overall nature of the flow for jet impingement onto the four porous screens used here. Firstly, if the screen is sufficiently open $(\beta>0.55$, say) the jet proceeds uniformly through the screen which acts mainly to enhance its width locally but reduce its momentum flux - the latter because of the screen drag. The increase in jet width is an inevitable consequence of the well-known process perhaps first identified by Taylor \& Batchelor (1949), who showed that screens placed normal to a flow tend to reduce departures from non-uniformity - which is of course why (generally wire-gauze) screens are used in wind tunnel settling chambers. In the present case, the upstream jet provides an unusually extreme case of mean-flow nonuniformity and the screen certainly reduces this substantially.

A curious feature for these more open screens is the apparently very small jet growth rate further downstream, which implies a large reduction in entrainment rate. Since the entrainment in free jets is mainly governed by the large eddies, the implication is that the screen tends to remove these large eddies preferentially. To check this conclusion a few spectral measurements were made at $x / H=1.3$. These did not extend to high frequencies; they were limited in that respect by the sampling rate of the LDA system - $40 \mathrm{~Hz}$ for these measurements, so no information relating to time scales shorter than $0.05 \mathrm{~s}$ was available. However, they were sufficient to allow integral scales to be estimated, on the basis that the integral scale, $L$, can be expressed in terms of the spectral energy at zero frequency, invoking Taylor's hypothesis. The usual relationship was used, i.e. $L=S(0) U / 4 u^{\prime 2}$, with $S(0)$ being the asymptotic spectral density at zero frequency, $u^{\prime 2}$ the total fluctuating energy and $U$ a convection speed, taken to be the local velocity. For the free jet $L_{x} / b$ was found to be 0.39 . This compares well with the Everitt \& Robins' (1978) result - 0.4. The lateral integral scale, $L_{y} / b$, was 0.26 . Corresponding values of $\left(L_{x} / b, L_{y} / b\right)$ for the $\beta=0.65,0.57$ and 0.5 cases were $(0.29,0.14),(0.18,0.11)$ and $(0.07,0.05)$, respectively. These results clearly confirm that the screens produce a substantial reduction in large eddy scales (compared with local jet width). In the present experiments measurements extended to $x / H=1.7$ at most and, since this represents only about three jet widths downstream of the screen, no conclusions are possible regarding jet development in the far field. In principle, presumably, the usual large-eddy structure would re-establish itself and the growth rate would thus increase again, but a rather different scale (and geometry) of experimental rig would be needed to investigate this point.

It is of note that for the $\beta=0.5$ screen there was no evidence of periodic (or quasiperiodic) instabilities detectable in the spectral data. This is interesting because it is well known that, in general, screens having open area ratios below about 0.57 tend to produce instabilities and non-uniformities (Bradshaw, 1966) because of preferential coelescence between the small jets issuing from adjacent holes in the screen. Neither were such nonuniformities detected in the mean flow data, even for the $\beta=0.41$ screen. This is not to say that they did not exist; they may have been sufficiently small-scale, compared with the 
underlying non-uniformity of the jet flow, to remain undetected, although we emphasise that no specific search for them was made.

Secondly, for sufficiently low porosity (below $\beta=0.5$ ) the jet impingement is similar to the case of a solid wall, so that wall jets appear on the upstream side of the screen. However, they also appear on the downstream side; this is probably not directly because of any boundary coupling from one side of the screen to the other, but rather because the centreline pressure just downstream of the screen remains high enough to provide the necessary forcing. In this case it seems that far downstream there is consequently an entrainment velocity in the negative $x$-direction. Details of the near-surface flows in the wall jets on either side of the screen have not been studied so there remain questions concerning how (or if) the wall jet on one side is affected by details of the jet on the other side. Such details will probably depend on the nature of the porous geometry, for even if the scale of the latter is small compared to the free jet width (at the screen location but in its absence) it may not be small compared to the inner region of the wall jet.

Thirdly, at intermediate porosities, around $\beta=0.5$, wall jets on the upstream and downstream sides of the screen seem to begin but they dissipate rapidly, with both velocity components falling close to zero quite quickly. Most of the volume flux of the original jet (plus its entrained fluid) appears in the jet continuing in the axial direction downstream of the screen. The upstream wall jet in this case is not sufficiently strong to prevent the entrainment processes leading to a clear recirculating region between it and the upstream normal jet. It is unclear whether or not it is significant that this intermediate range of $\beta$ coincides roughly with the point at which smooth downstream flow behind wire gauze screens is known to break down to become unstable and contain irregular non-uniformities - around $\beta=0.57$, as mentioned above. This point requires further study and would be important if, for the even lower $\beta$ range, the structure of the wall jets turns out to depend significantly on the geometry of the porosity (for fixed $\beta$ ). If this were the case, as it surely must be if the porosity scale is not small compared to the jet scale, one would expect the $\beta$ value at which the qualitative change in flow structure occurs to be dependent on details of the porosity geometry. This is obviously a much more difficult flow to consider.

\section{Conclusions}

The results presented above have shown how the nature of the mean flow of a planar turbulent jet impinging normally onto a porous wall depends strongly on the particular value of the posority. We have demonstrated that if the porosity is low enough wall jets appear on both sides of the screen and this can lead to an 'upstream' entrainment flow in the far field downstream of the screen. For higher porosities such wall jets do not exist (although in an intermediate porosity range they may begin but then disappear rapidly). The jet in this higher porosity case expands significantly on passing through the screen and thereafter seems to grow only very slowly, if at all, because (apparently) of the destruction of the large-eddy structures. It is emphasised again that this is a preliminary investigation. There remain various questions, which can only be properly answered by a detailed study of the turbulence; this is now under way. It would also be interesting, and perhaps even more useful from a practical perspective, to study the corresponding axi-symmetric case.

Impinging jet flows have been a particular problem for turbulence modellers over many years. Most of the effort has been concentrated on radial jets and it has become quite clear that standard closure models, even second order ones, are not adequate for such flows. Particular attention has to be paid to the wall-reflection terms that appear in the modelled equations but it has been shown that if this is done, second moment closures or even higher 
order eddy viscosity models can achieve some success (e.g. Craft \& Launder, 1996, Craft et al, 1997). Flows including impingement onto porous surfaces have received much less attention. The porosity models that are often part of commercially available CFD packages (and are usually based on variants of Darcy's law) would be inappropriate for the present case. So we conclude by pointing out that the flows described in this paper would provide challenging test cases for numerical modellers.

\section{Acknowledgements}

The work could not have been completed without the assistance of the technical staff of the EnFlo laboratory. We thank, in particular, Mr Tom Lawton and Mr Allan Wells for their cheerful and unstinting help. RC is grateful to Silsoe Research Institute and to the Engineering \& Physical Sciences Research Council, who funded his Engineeering Doctorate programme during which the experiments were undertaken.

\section{$7 \quad$ References}

BELTAOS S \& RAJARATNAM N (1973) Plane turbulent impinging jets. J. Hyd. Res. 11, 29-59.

BRADSHAW P (1965) The effect of wind tunnel screens on nominally two-dimensional boundary layers. J. Fluid Mech. 22, 679-687.

CRAFT T \& LAUNDER BE (1996) A Reynolds stress closure designed for complex geometries. Int. J. Heat and Fluid Flow, 17, 245-254.

CRAFT T, LAUNDER BE \& SUGA K (1997) Prediction of turbulent transitional phenomena with a non-linear eddy-viscosity model. Int. J. Heat and Fluid Flow, 18, 15-28.

CANT RJ (1999) Air jet behaviour in porous structures with application to orchard spraying. EngD Thesis, University of Surrey, Guildford.

EVERITT KW \& ROBINS AG (1978) The development and structure of plane turbulent jets. J. Fluid Mech. 88, 563-583.

FLORA JJ \& GOLDSCHMIDT VW (1969) Virtual origins of a free plane turbulent jet. AIAA Journal, 7, 2344-2346.

FOSS JF (1979) Measurements in large-angle oblique jet impingement flow. AIAA J. 17, 801-802.

GEORGE WK \& TAULBEE DB (1992) Designing experiments to test closure hypotheses. Experimental Thermal \& Fluid Science, 5, 249-259.

GURIDI I (1997) General characteristics of a free and impinging planar jet. Report MEFD/97.70, Department of Mechanical Engineering, University of Surrey, 96pp.

GUTMARK E \& WYGNANSKI I (1976) The planar turbulent jet. J. Fluid Mech. 73, 465-495.

HUSSAIN AKMF \& CLARK AR (1977) Upstream influence on the near field of a plane turbulent jet. Phys. of Fluids, 20, 1416-1426.

LOONEY MK \& WALSH JJ (1984) Mean flow and turbulent characteristics of free and impinging jet flows. J. Fluid Mech. 147, 397-429.

ÖZDEMIR IB \& WHITELAW JH (1992) Impingement of an axisymmetric jet on unheated and heated flat plates. J. Fluid Mech., 240, 503-532.

SCHNEIDER W (1985) Decay of momentum flux in submerged jets. J. Fluid Mech. 154, 91-110.

TAYLOR GI \& BATCHELOR GK (1949) Quart. J. Mech. Appl. Math. 2, 1- 
WALKLATE PJ, WEINER KL \& PARKIN CS (1996) Analysis of and experimental measurements made on a moving air-assisted sprayer with two-dimensional air-jets penetrating a uniform canopy. J. Agr. Eng. Res. 63, 365-378.

WEINER KL (1993) Air jet behaviour in crop like structures. PhD Thesis, Cranfield University, Bedfordshire, 163pp.

WYGNANSKI I, KATZ Y \& HOREV E (1992) On the applicability of various scaling laws to the turbulent wall jet. J. Fluid Mech. 234, 669-690. 